\title{
A Multiscale Feature Detector for Morphological Analysis of the Brain
}

\author{
Marius George Linguraru, Miguel Ángel González Ballester, and Nicholas Ayache \\ Epidaure Research Project, INRIA, 2004 route des Lucioles, B.P. 93 \\ 06902 Sophia Antipolis Cedex, France \\ \{Marius.Linguraru, Miguel.Gonzalez, \\ Nicholas.Ayache\} @sophia.inria.fr
}

\begin{abstract}
Feature detection on MR images has largely relied on intensity classification and gradient-based magnitudes. In this paper, we propose the use of phase congruency as a more robust detection method, as it is based on a multiscale intensity-invariant measure. We show the application of phase congruency for the detection of cortical sulci from T2 weighted MRI. Sulci represent important landmarks in the structural analysis of the brain, as their location and orientation provide valuable information for diagnosis and surgical planning. Results show that phase congruency outperforms previous techniques, even in the presence of intensity bias fields due to magnetic field inhomogeneity.
\end{abstract}

\section{Introduction}

The anatomical variability of the cortical surface is a topic of great interest, as the location and orientation of cortical sulci have been related to clinical conditions such as schizophrenia [2]. Additionally, sulci can be used as reference to locate internal brain structures and thus plan an intervention [9]. This makes the construction of statistical atlases [11] for the identification of the main sulci an active area of research [1]. Finally, sulci need to be identified to remove false positives in tumour or multiple sclerosis lesion detection.

It is generally accepted that T2-weighted MRI provides good detection of cortical geometry, as it depicts clearly the contrast between the cortical grey matter and the cerebrospinal fluid surrounding it. This paper presents the results of a set of experiments performed in order to delineate sulci from T2 MRI. It will be shown that classical approaches based on intensity thresholding or gradient-based edge detection [4] often pose problems due to intensity variations across the image and smooth tissue transitions due to partial volume effects. The same applies to more sophisticated methods, such as geometry-driven diffusion [2], where the image intensity still plays a key role. An alternative algorithm extracts the crest lines with positive largest curvature, which can be interpreted as sulci in MR images [10]. Only the bottom of sulci would be detected by such an approach. Level sets have also provided promising results in [13], but the method requires user initialisation and a long processing time. 
In 1987, the concepts of local energy and phase congruency were introduced as a new intensity-invariant feature detector that performs well even in areas of low contrast [7]. This technique was adopted in our method and will be described in the next section.

Section 3 expends on the reasoning behind the segmentation of sulci and section 4 shows results. We also show examples of the use of intensity thresholding and gradient-based edge detection on a typical T2 weighted image and highlight the problems encountered. Our approach is particularly adapted to the presence of intensity variations due to inhomogeneity of the magnetic field in the MRI machine; this is shown in section 5. Finally, section 6 provides discussion and directions of future work.

\section{Local Energy and Phase Congruency}

Morrone and Owens [7] and later Kovesi [3] noted that local energy points out changes in the shape of a signal. Likewise, step edges, ramps or more complex intensity variations correspond to signal locations at which the local Fourier components are all in phase or points of maximum phase congruence. Let $f(x)$ be a one-dimensional signal. It can be reconstructed from its Fourier spectrum by:

$$
f(x)=\int_{-\infty}^{\infty} a_{\omega} \cos \left(\omega x+\phi_{\omega}\right) d \omega,
$$

where $a_{\omega}$ is the amplitude and $\omega x+\phi_{\omega}$ is the phase offset. Phase congruency is a normalised measure of local energy, which makes it invariant to the image contrast and brightness. Computing the value of local energy, local maxima correspond to feature points where phase congruency is maximum. Therefore, a feature is located at a point where the phase congruency, a scalar measure with values in between 0 and 1 , is high.

The local energy can be obtained from the amplitude of the analytic wavelet transform (2), a convolution of the signal with a pair of quadrature filters, for which we use log-Gabor functions. The log-Gabor function is defined in the frequency domain as in Equation (3).

$$
\begin{gathered}
L E=|\langle f, W\rangle| \text {, where } W \text { is an analytic wavelet } \\
G(\omega)=e^{\frac{-\left(\log \left(\omega / \omega_{0}\right)\right)^{2}}{2\left(\log \left(\kappa / \omega_{0}\right)\right)^{2}}} \text {, if } \omega>0 \text {, and zero otherwise, }
\end{gathered}
$$

where $\omega_{\mathrm{o}}$ is the filter's centre frequency. The term $\kappa / \omega_{\mathrm{o}}$ is held constant according to the desired number of octaves of the filter bandwidth. Phase congruency (PC) is then computed from the local energy (LE), as below:

$$
P C(x)=\frac{L E(x)}{\int_{-\infty}^{\infty} a_{\omega} d \omega}
$$


Combining the phase information with the phase congruency or local energy, it is possible to distinguish between peaks, valleys, upsteps and downsteps. The local energy and phase congruency computation can be extended to $2 \mathrm{D}$ if it is assumed that the signals of interest have simple neighbourhoods, that is, vary locally only in one direction. This allows the interpolation of local energy and estimation of orientation from a minimum of three energy outputs obtained from three symmetrical distributed directions: $30^{\circ}, 90^{\circ}$ and $150^{\circ}$. The energy is computed in each orientation and extended with a spread function. In practice, six directions $\left(0^{\circ}, 30^{\circ}, 60^{\circ}, 90^{\circ}, 120^{\circ}\right.$ and $150^{\circ}$ ) were used to accommodate the complexity of the images.

Phase congruency can be applied to an image at multiple scales and at different filter bandwidths. We can choose the most suitable scale and bandwidth to do a single scale analysis of phase congruency or local energy, or multiscale analysis using Kovesi's method [3].

\section{Sulci Extraction}

The cerebrospinal fluid surrounds the brain and fills in the cortical sulci. In T2 weighted MR images this fluid shows bright intensities, in contrast to the darker intensities of the cortical grey matter. However, this contrast is highly reduced by partial volume effects, making it often difficult to delineate sulcal lines.

Sulcal patterns have certain characteristics that make them particularly suited for the application of phase congruency:

- $\quad$ sulci are thin anatomical structures that appear as small bright lines in T2 weighted MR images;

- sulci are locally linear high-frequency structures with well-defined orientation;

- the orientation of sulci gives valuable information in the qualitative analysis of the brain.

The method we propose for sulci extraction is based on the local energy model for feature detection of Kovesi [3]. The principles behind local energy and phase congruency methods have been presented above.

\section{Results}

In this section we show the results we obtained on feature segmentation of typical T2 images of the brain. We compare the performance of our approach over classical methods.

Basic feature detectors in image processing imply thresholding and the computation of gradient. While thresholding is global and expected to give poor results in the segmentation of highly variable structures, such as sulci, image gradient is a local measure with higher sensitivity to regional intensity variations. From the gradient value, an edge detector can be built that will be sensitive to image contrast, which overlooks the less prominent intensity changes. 
The major contribution of phase congruency is its invariability to intensity parameters. This makes it a better detector, and for the right combination of scales (i.e. wavelength and noise barrier) we detect accurately the sulci as linear structures with high contrast in the image. Figure 1 shows the comparative results obtained using thresholding, gradient and phase congruency. The example shown uses a T2 Fast Spin Echo image data set with a resolution of 256x256x64 and a voxel size of $0.94 \mathrm{~mm} \times 0.94 \mathrm{~mm} \times 2 \mathrm{~mm}$. We note the better performance of the local energy-based approach.

In a similar manner to Kovesi [3], a noise estimation procedure is employed in order to eliminate from the phase congruency response the points of lower energy for a less textured result. A small wavelength combined with a high noise threshold have given the desired results.
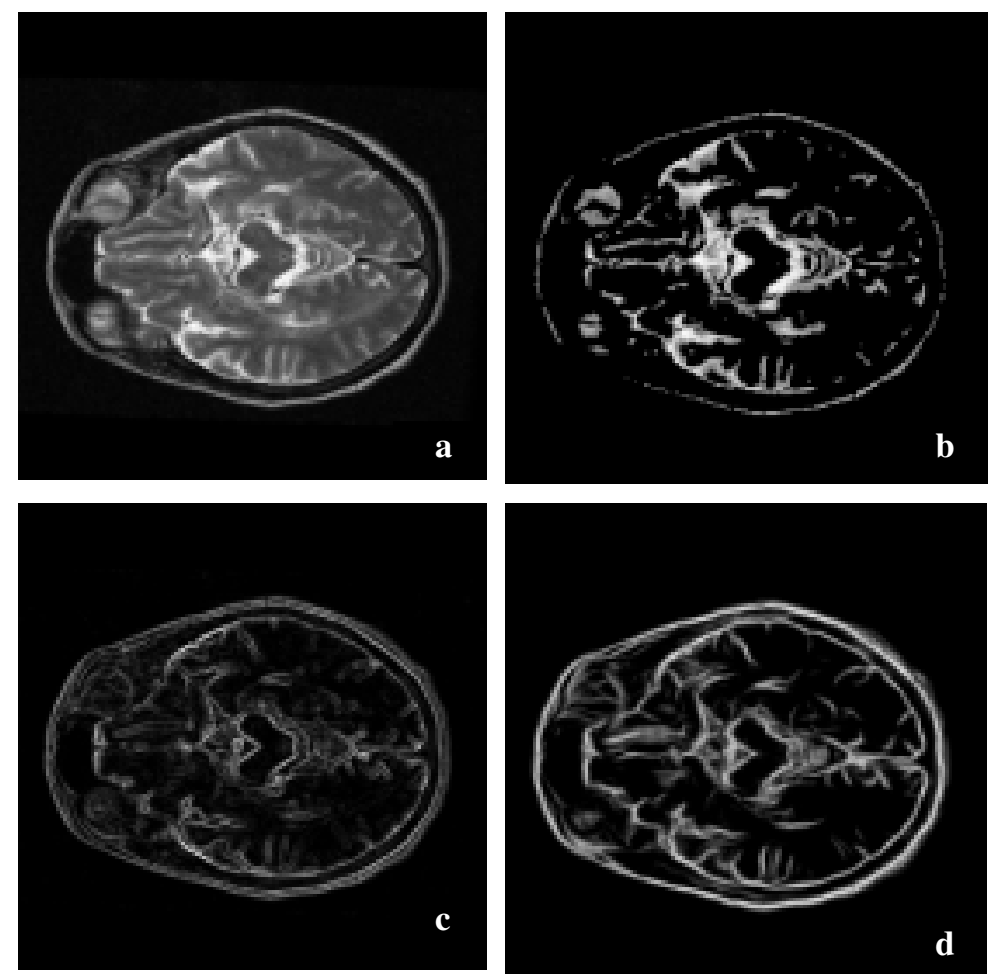

Fig. 1. Comparative results of feature detection on a T2-weighted image data set; (a) the original MR slice; (b) results using thresholding; (c) results using a gradient-based edge detector; (d) feature extraction using phase congruency.

The method we present is applied to 2D MRI slices of the brain. Since MR data is $3 \mathrm{D}$, an appropriate extension of the algorithm to the third dimension is desirable, and will be tackled in future work. For the time being, we found that the current 2D method performs very well in the sense that it is consistent across slices, as shown in Figures 2 and 3. 

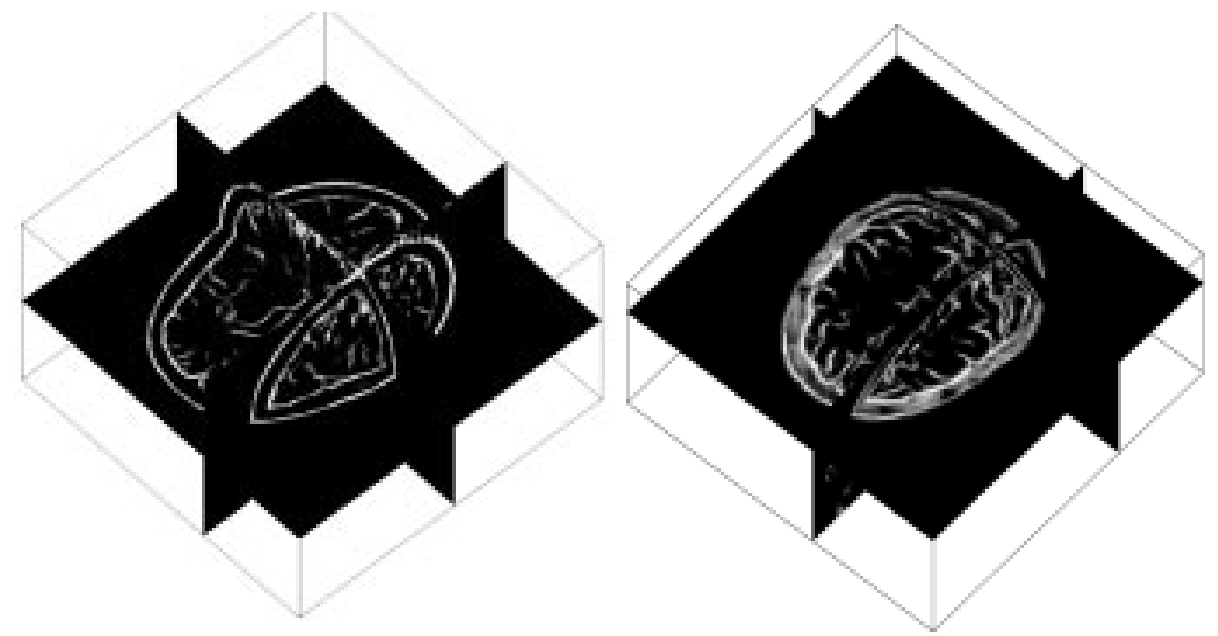

Fig. 2. The 3D volume of detected features on two 256x256x64 T2-weighted MR images.

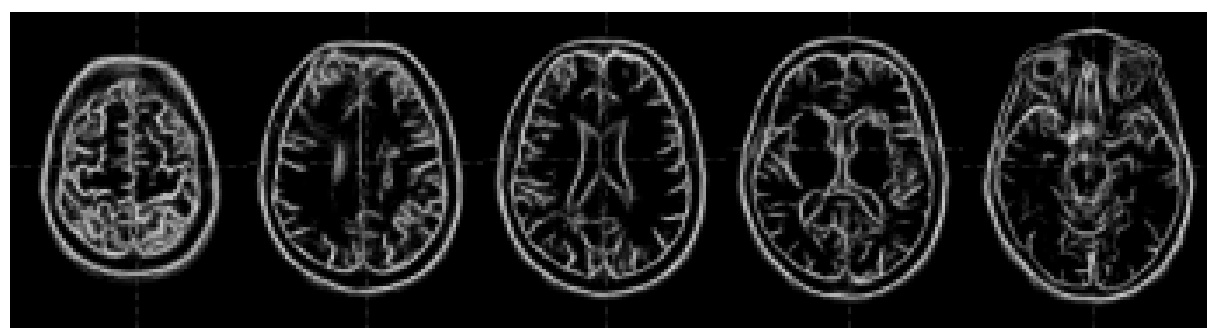

Fig. 3. Detection results over five MR image slices.

\section{Magnetic Field Inhomogeneity}

The intensity-invariant nature of phase congruency is particularly relevant in the presence of intensity variations across the data set. This is the case in MRI when the magnetic field in the region of interest is not completely uniform. Several techniques have been proposed in order to correct for this position-dependent biases, but they mostly rely on obtaining good estimates of typical tissue intensities [5,12], or alternatively are simplistic models based on removing the lower frequency components of the Fourier decomposition of the image [6]. Our approach does not require the time consuming step of bias field correction due to the properties of phase congruency.

We prove the usefulness of phase congruency as an edge detector in the presence of bias fields by comparing its performance with classical approaches. For the sake of clarity in the comparison of results, the data set used for the results reported above (Figure 1) is reused, this time corrupted by a synthetically-generated bias field. This 
bias field is simply a linear function of the $\mathrm{x}$ and $\mathrm{y}$ location in the image (Figure 4.a). The corrupted image is shown in Figure 4.b.

Thresholding or intensity-based classification are highly affected by this bias (Figure 4.c). Gradient-based edge detectors are affected in the sense that setting a global threshold on gradient strength becomes more difficult, as intensity differences also vary locally. On the contrary, phase congruency results seem very robust to the presence of contrast variations across the image, as shown in Figure 4.e.
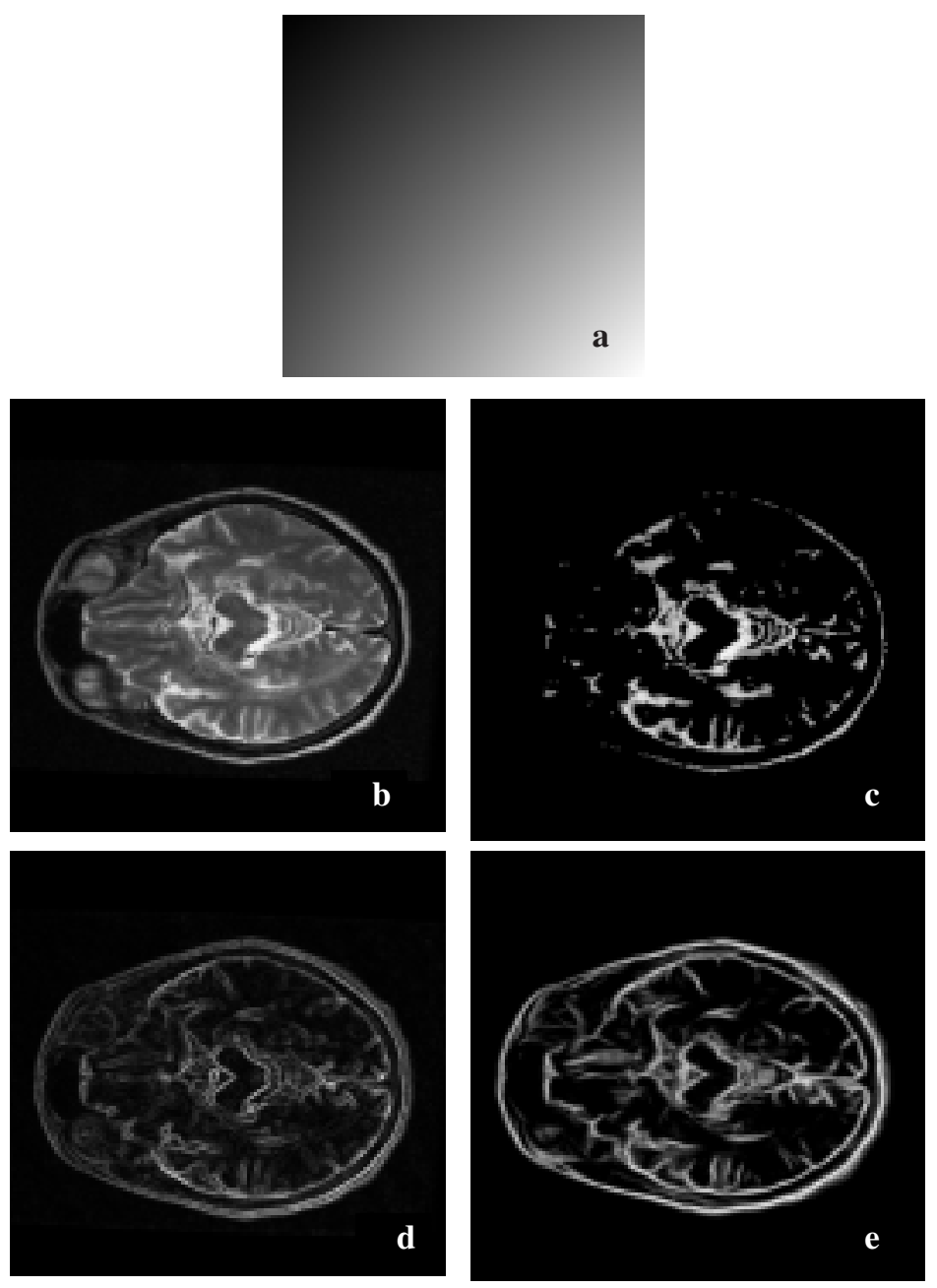

Fig. 4. Comparative results of feature detection after applying a bias field; (a) the syntheticallygenerated bias field that we used to corrupt the image data set; (b) the corrupted image; (c) results using thresholding; (d) results using gradient; (e) results using phase congruency. 


\section{Discussion and Future Work}

We presented a method for feature extraction for brain morphological studies. Using phase congruency, the detection results are not sensitive to image intensity and overcome common difficulties in brain imaging, such as the presence of a bias field. The method outperforms thresholding and gradient-based segmentation approaches and provides a good localisation of features.

In addition to feature localisation by local energy, phase can be used to classify features relative to their dominant orientation (Figure 5). The orientation of the filter which gives the maximum response across all scales is kept. Empirically, we found good practical results by setting the number of orientations to six over four scales. At each pixel of a sulcus, the scale that has the largest magnitude coefficients is selected to approximate the structure. Efficiently, this results in a combination of the multiscale information.

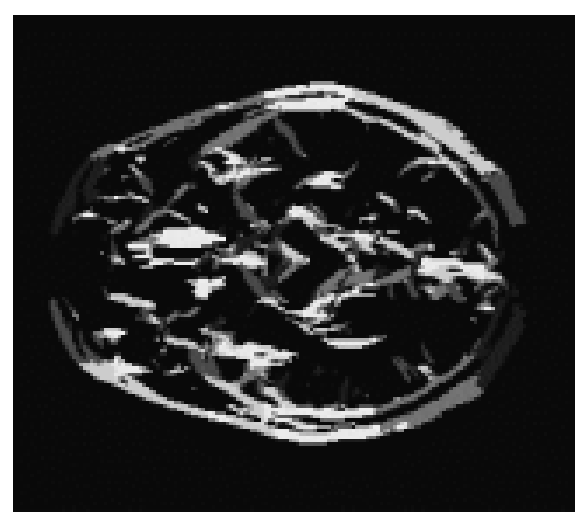

Fig. 5. The orientation map of the detected features. We used 6 different orientations coded in distinct grey-levels in the map.

A noise estimation procedure is employed in order to filter out responses at high frequencies [3]. We have found that the performance of Kovesi's method depends on the settings of this noise estimation process. The range of scales to be considered and noise parameters were set empirically for a good sulci detection.

The implementation of phase congruency used for the results in this paper is 2D. An extension to 3D, based on the application of filters at orientations sampled from a unit sphere [8] will be used in the future. A pure 3D scheme will result in more information about dominant orientations of sulci crossing image slices, as well as a more robust noise estimation, performed over the whole volume. The computation time on a 256x256x64 MR image is of approximately six minutes on a Pentium III machine with $1 \mathrm{~GB}$ RAM and $1 \mathrm{GHz}$ before the optimisation of the code.

Future applications of the method will focus on the detection of evolving tumours and multiple sclerosis lesions from temporal sequences of MR images. Sulci will be detected as structures with minimal temporal variations, in order to remove false positives. 


\section{References}

1. Le Goualher, G. Collins, D.L. Barillot, C. Evans, A.C.: Automatic Identification of Cortical Sulci using a 3D Probabilistic Atlas. In Wells, W.M. et al. (Eds): MICCAI'98, LNCS 1496, Springer-Verlag Berlin Heidelberg (1998) 509-518

2. Kikinis, R. Shenton, M.E. Gerig, G. Hokama, H. Haimson, J. O'Donnell, B.F. Wible, C.G. McCarley, R.W. Jolesz, F.A.: Temporal Lobe Sulco-Gyral Pattern Anomalies in Schizophrenia: An in Vivo MR Three-Dimensional Surface Rendering Study. In: Neuroscience Letters, Vol. 182 (1994) 7-12

3. Kovesi, P.: Image Features from Phase Congruency. In: Videre: Journal of Computer Vision Research, Vol. I (1999) 1-26

4. Kruggel, F. Horsch, A. Mittelhäußer, G. Schnabel, M.: Image Processing in the Neurologic Sciences. In: Proc. of the IEEE Workshop on Biomedical Image Analysis, IEEE Computer Society Press Washington (1994) 214-223

5. Van Leemput, K. Maes, F. Vandermeulen, D. Colchester, A. Suetens, P.: Automated Segmentation of Multiple Sclerosis Lesions by Model Outlier Detection. In: IEEE Transactions on Medical Imaging, Vol. 20(8) (2001) 677-688

6. Listerud, J. Lenkisnki, R.E. Kressel, H.Y Axel, L.: The Correction of Nonuniform Signal Intensity Profiles in Magnetic Resonance Imaging. In: Journal of Digital Imaging, Vol. 2(1) (1989) 2-8

7. Morrone, M.C. Owens, R.A.: Feature Detection from Local Energy. In: Pattern Recognition Letters, Vol. 6 (1987) 303-313

8. Mulet-Parada M., Noble, J.A.: 2D+T Acoustic Boundary Detection in Echocardiography. In Wells, W.M. et al. (Eds.) MICCAI'98, LNCS 1496, Springer-Verlag Berlin Heidelberg (1998) 806-813

9. Nakajima, S. Kikinis, R. Jolesz, F.A. Atsumi, H. Leventon, M.E. Grimson, W.E.L. Hata, N. Metcalf, D.C. Moriarty, T.M. Black, P.M. Garada, B. Alexander, E.: 3D MRI Reconstruction for Surgical Planning and Guidance. In: Alexander, E. MacIunas, R.J. (Eds) Advanced Neurosurgical Navigation, Thieme Medical Pub. (1998) 137-145

10. Pennec, X. Ayache, N. Thirion, J.P.: Landmark-Based Registration Using Features Identified trough Differential Geometry. In Bankman, I. (Ed.) Handbook of Medical Imaging - Processing and Analysis, Chapter 31, Academic Press (2000) 499-513

11. Thompson, P.M. Mega, M.S. Narr, K.L. Sowell, E.R. Blanton, R.E. Toga, A.W.: Brain Image Analysis and Atlas Construction. In Sonka, M. Fitzpatrick, J.M. (Eds.) Handbook of Medical Imaging Vol. 2 - Medical Image Processing and Analysis, Chapter 17, SPIE Press, Vol. PM80 (2000)

12. Wells, W.M. Grimson, W.E.L. Kikinis, R. Jolesz, F.A.: Adaptive Segmentation of MRI Data. In: IEEE Transactions on Medical Imaging, Vol. 15(4) (1996) 429-442

13. Zeng, X. Staib, L.H. Schultz, R.T. Duncan, J.S.: Segmentation and Measurement of the Cortex from 3D MR Images. In Wells, W.M. et al. (Eds.) MICCAI'98, LNCS 1496, Springer-Verlag Berlin Heidelberg (1998) 519-530 